DOCUMENTOS OCASIONALES

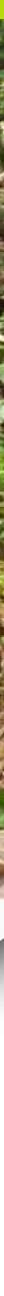

\title{
¿Qué sabemos sobre las turberas peruanas?
}

Mariela López Gonzales

Kristell Hergoualc'h

Óscar Angulo Núñez

Timothy Baker

Rodney Chimner

Jhon del Águila Pasquel

Dennis del Castillo Torres
Luis Freitas Alvarado

Beatriz Fuentealba Durand

Erick García Gonzales

Eurídice Honorio Coronado

Hirose Kazuyo

Erik Lilleskov

Natalia Málaga Durán
Mónica Maldonado Fonkén

Manuel Martín Brañas

Tony Mori Vargas

Ana María Planas Clarke

Katherine Roucoux

Faustino Vacalla Ochoa

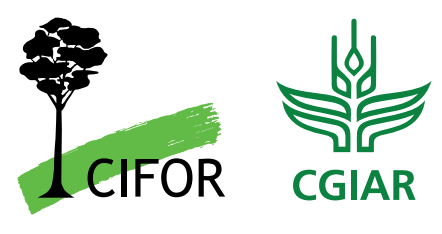





\section{¿Qué sabemos sobre las turberas peruanas?}

Mariela López Gonzales

Centro para la Investigación Forestal Internacional (CIFOR)

Kristell Hergoualc'h

Centro para la Investigación Forestal Internacional (CIFOR)

\section{Óscar Angulo Núñez}

Instituto de Ciencias de la Naturaleza, Territorio y Energías Renovables de la Pontificia Universidad Católica del Perú (INTE-PUCP)

\section{Timothy Baker \\ University of Leeds}

\section{Rodney Chimner}

School of Forest Resources and Environmental Science, Michigan Technological University

\section{Jhon del Águila Pasquel}

Instituto de Investigaciones de la Amazonía Peruana (IIAP)

\section{Dennis del Castillo Torres}

Instituto de Investigaciones de la Amazonía Peruana (IIAP)

\section{Luis Freitas Alvarado}

Instituto de Investigaciones de la Amazonía Peruana (IIAP)

\section{Beatriz Fuentealba Durand}

Dirección de Ecosistemas de Montaña, Instituto Nacional de Investigación en Glaciares y Ecosistemas de Montaña (INAIGEM)

\section{Erick García Gonzales}

Dirección de Calidad y Evaluación de Recursos Hídricos, Autoridad Nacional del Agua (ANA)

\section{Eurídice Honorio Coronado}

Instituto de Investigaciones de la Amazonía Peruana (IIAP)

Hirose Kazuyo
Japan Space Systems

Erik Lilleskov

USDA Forest Service, Northern Research Station

Natalia Málaga Durán

Centro para la Investigación Forestal Internacional (CIFOR)

\section{Mónica Maldonado Fonkén}

División de Ecología Vegetal, Centro de Ornitología y

Biodiversidad (CORBIDI)

\section{Manuel Martín Brañas}

Instituto de Investigaciones de la Amazonía Peruana (IIAP)

\section{Tony Mori Vargas}

Autoridad Regional Ambiental, Gobierno Regional de Loreto (ARA-GOREL)

\section{Ana María Planas Clarke SilvaCarbon}

Katherine Roucoux University of St Andrews

\section{Faustino Vacalla Ochoa \\ Programa Forestal SERFOR-CAF del Servicio Nacional Forestal y de Fauna Silvestre (SERFOR)}


Documentos Ocasionales 208

(c) 2020 Centro para la Investigación Forestal Internacional (CIFOR)

(c) (1) LY (CC BY 4.0), http://creativecommons.org/licenses/by/4.0/
(Cy

ISBN 978-602-387-141-4

DOI: $10.17528 /$ cifor/007847

López Gonzales M, Hergoualc'h K, Angulo Núñez Ó, Baker T, Chimner R, del Águila Pasquel J, del Castillo Torres D, Freitas Alvarado L, Fuentealba Durand B, García Gonzales E et al. 2020. ¿Qué sabemos sobre las turberas peruanas? Documentos Ocasionales 208. Bogor, Indonesia: CIFOR.

Colaboradores: José Álvarez Alonso (Dirección General de Diversidad Biológica, Ministerio del Ambiente - MINAM); Janet Calvo Vargas (Dirección General de Diversidad Biológica, MINAM); Claudia Enrique Fernández (Dirección de Conservación Sostenible de Ecosistemas y Especies, MINAM); Walter Huamaní (Dirección General de Diversidad Biológica, MINAM); y Tatiana Pequeño Saco (Dirección de Monitoreo y Evaluación de Recursos Naturales del Territorio, MINAM)

Foto de portada por Kristell Hergoualc'h/CIFOR

Muestreo de Gases de Efecto Invernadero en turberas de aguajales de la Amazonía peruana (Reserva regional de Quistococha, Loreto-Perú).

\author{
CIFOR \\ Jl. CIFOR, Situ Gede \\ Bogor Barat 16115 \\ Indonesia \\ $\mathrm{T}+62$ (251) 8622-622 \\ $F+62$ (251) 8622-100 \\ E cifor@cgiar.org
}

\title{
cifor.org
}

Quisiéramos agradecer a todos los socios financieros que apoyaron esta investigación a través de sus contribuciones al Fondo de CGIAR. Para ver la lista de donantes del Fondo, visite: http://www.cgiar.org/our-funders/

Cualquier opinión vertida en este documento es de los autores. No refleja necesariamente las opiniones de CIFOR, de las instituciones para las que los autores trabajan o de los financiadores. 


\section{Contenido}

Agradecimientos $\quad$ iv

1 Introducción 1

2 Estado de la cuestión $\quad 2$

2.1 Importancia de las turberas peruanas 2

2.2 Amenazas 5

$\begin{array}{lll}2.3 & \text { Marco normativo } & 7\end{array}$

3 Oportunidades y retos 9

4 Recomendaciones $\quad 12$

$\begin{array}{ll}\text { Referencias } & 13\end{array}$

\section{Lista de tablas y figuras}

\section{Tablas}

1 Área de turberas estimada y ecosistemas con potencial de formación de turba, por región del país

$2 \quad$ Principales causas de la degradación de las turberas en el Perú 5

3 Objetivos y necesidades de investigación científica para la protección y el manejo sostenible de las turberas peruanas

\section{Figura}

1 Ecosistemas con potencial de formación de turba en el Perú 


\section{Agradecimientos}

Esta investigación se realizó en el marco del Programa de Adaptación y Mitigación Sostenibles de los Humedales (SWAMP, por sus siglas en inglés) y el Estudio Comparativo Global sobre REDD+ (GCS-REDD+) de CIFOR. Recibió el generoso apoyo de los Gobiernos de los Estados Unidos de América (Subvención MTO-069018) y Noruega (Subvención QZA-12/0882). Se llevó a cabo como parte del Programa de Investigación del CGIAR sobre Bosques, Ârboles y Agroforestería (CRP-FTA, por sus siglas en inglés), con el apoyo financiero de los donantes del Fondo del CGIAR.
Los autores están sumamente agradecidos con todos los participantes del taller "Avances y desafíos para el manejo sostenible de las turberas de aguajales en la Amazonía" (Iquitos, octubre de 2018) y el Simposio Nacional sobre la Gestión Sostenible de las Turberas Peruanas (Lima, abril de 2019) por sus valiosos aportes a las discusiones. Esta publicación ha seguido un procedimiento de revisión por pares, por lo que agradecemos las valiosas revisiones y sugerencias de Rodolfo Iturraspe, Verónica Pancotto, Juan Carlos Benavides, Christopher Martius y Peter Cronkleton. 


\section{Introducción}

Las turberas son humedales donde la deficiencia de oxígeno que resulta de un nivel alto de la capa freática permite la descomposición lenta de la vegetación muerta in situ y la formación de turba. Los suelos turbosos se distinguen de los suelos minerales por tener un alto contenido de carbono $(\mathrm{C})$ y un espesor de materia orgánica parcialmente descompuesta — es decir, la turbade hasta varios metros de profundidad. Aunque las turberas ocupan solo el 2,8 \% de la superficie terrestre (Xu et al. 2018), se estima que almacenan hasta 850 gigatoneladas $\left(1 \mathrm{Gt}=10^{15} \mathrm{~g}\right)$ de $\mathrm{C}$ en el suelo (Yu et al. 2012; Gumbricht et al. 2017); es decir, más de cuatro veces las reservas de $\mathrm{C}$ de la vegetación por encima del suelo en todo el trópico (188 Gt C; Avitabile et al. [2015]). Debido al importante rol que cumplen las turberas en la fijación y el almacenamiento de $\mathrm{C}$, su conservación, manejo sostenible y restauración son considerados claves entre las soluciones climáticas naturales para lograr el objetivo del Acuerdo de París de mantener el calentamiento global por debajo de $2{ }^{\circ} \mathrm{C}$ (Griscom et al. 2017). Por otra parte, las turberas son esenciales para la adaptación al cambio climático mediante su provisión de servicios ecosistémicos relacionados con el agua, como el almacenamiento y la regulación hídrica, la amortiguación frente a inundaciones y la purificación del agua a escala local y regional. También constituyen el hábitat de una biodiversidad única y desempeñan importantes funciones económicas y culturales para el bienestar humano. Sin embargo, son ecosistemas muy frágiles que se han desarrollado durante miles de ańos, desde las últimas glaciaciones, y cuya degradación ocasiona pérdidas irreparables y emisiones de gases de efecto invernadero (GEI) desproporcionadamente altas (Gaveau et al. 2014; Hergoualc'h et al. 2018).

En el trópico, las mayores áreas de turberas se encuentran en Indonesia, en la cuenca del río Congo y en la cuenca del río Amazonas (Gumbricht et al. 2017). Durante décadas, los bosques pantanosos de turba del sudeste asiático (Indonesia y Malasia) han sufrido una destrucción masiva, debido sobre todo a cambios en el uso de la tierra para favorecer cultivos agrícolas o plantaciones, lo que normalmente implica el drenaje de la turbera como actividad inicial. Se estima que, entre 1990 y 2010, se ha perdido el $50 \%$ de estos bosques (Miettinen et al. 2012). En contraste, las turberas peruanas están menos degradadas que las del sudeste asiático (Draper et al. 2014), pero podrían ir rumbo a un destino similar en el futuro si no se aprende de lo ocurrido en otros países (Lilleskov et al. 2017). Por ello, resulta imprescindible que las políticas peruanas aseguren la conservación y el uso sostenible de las turberas y las prioricen dentro de la agenda nacional sobre el clima y el ambiente. A nivel internacional, existen varias iniciativas que reconocen la importancia de las turberas y que promueven su uso racional. Entre ellas, la Convención de Ramsar, a la que el Perú se adhirió en 1992, exhorta a las Partes a que otorguen un carácter prioritario a sus turberas (Ramsar 2002). Entre sus actividades de apoyo a las Partes, la Convención de Ramsar provee una guía para inventarios de turberas en el trópico (Ramsar 2018). También está la Iniciativa Global por las Turberas (GPI por sus siglas en inglés), cuyo objetivo es evaluar el estado y el manejo sostenible de las turberas, su importancia en las economías locales y nacionales, y su rol fundamental para el logro de las metas globales de mitigación del cambio climático. El Perú, además, ha sido invitado a formar parte del Centro Internacional de Turberas Tropicales (ITPC por sus siglas en inglés), una iniciativa liderada por Indonesia e integrada por países del trópico ricos en turberas, como la República Democrática del Congo y la República del Congo.

La presente publicación tiene como objetivo sintetizar los conocimientos actuales sobre las turberas peruanas, haciendo énfasis en las amenazas que ponen en riesgo su conservación, los retos que enfrentan y las oportunidades que se ofrecen para su gestión sostenible. 


\section{Estado de la cuestión}

\subsection{Importancia de las turberas peruanas}

Según estudios recientes, el Perú es uno de los países más ricos en turberas en el trópico (Gumbricht et al. 2017). Sus turberas se distribuyen a lo largo de las regiones amazónica, andina y costera, con una predominancia marcada en la Amazonía, según los mapas existentes (Gumbricht et al. 2017; Chimner et al. 2019) y las áreas de los ecosistemas identificadas con potencial de formación de turba (tabla 1, figura 1). En la Amazonía, más de tres cuartas partes de las turberas se encuentran hacia el norte, en el departamento de Loreto (Gumbricht et al. 2017). En las zonas altoandinas, la mayoría de las turberas se ubican a más de $3800 \mathrm{~m}$ de altura (Chimner et al. 2019). A la fecha, el Perú no dispone de un inventario nacional de turberas que permita cuantificar con exactitud su área total.

Las turberas amazónicas, llamadas localmente "chupaderas", albergan tres tipos principales de vegetación según Draper et al. (2014). Los de mayor extensión (78 \% del área de turberas estudiada) son los pantanos de palmeras, que se encuentran dominados por la palmera Mauritia flexuosa, más conocida en el Perú como aguaje. Los varillales hidromórficos (11\%) son bosques densos de árboles delgados de diversidad limitada, con algunas especies típicas de bosques de arena blanca (Draper et al. 2017). También de menor extensión (11\%) son los pantanos abiertos dominados por herbáceas y especies arbustivas. Además, Lähteenoja et al. (2012) reportaron la presencia de turba en un bosque inundado estacionalmente por aguas negras (o tahuampa) en Loreto. En zonas altoandinas, la turba suele estar presente en humedales llamados bofedales (Maldonado Fonkén 2014), en ecosistemas de jalcas localizados entre 3500 y $4000 \mathrm{~m}$ de altura (Cooper et al. 2010), en ecosistemas lacustres como totorales (Medrano, Chupan y Vila 2012) y en los páramos (MINAM [Ministerio del Ambiente del Perú] 2018b). Las turberas en las montañas se caracterizan por la predominancia de plantas que crecen formando cojines (p. ej., Distichia spp.),

Tabla 1. Área de turberas estimada y ecosistemas con potencial de formación de turba, por región del país

\begin{tabular}{lcl}
\hline Región & Área de turbera estimada $\left(\mathbf{k m}^{2}\right)$ & Ecosistema con potencial de formar turba \\
\hline Amazonía & $74644^{\#}$ & Pantano de palmeras \\
& & Varillal hidromórfico \\
& & Pantano herbáceo-arbustivo \\
& & Bosque aluvial inundable \\
\hline Andes & $13800^{\&}$ & Bofedal \\
& & Páramo \\
& & Jalca \\
\hline Costa & n. d. & Manglar \\
& & Humedal costero \\
\hline
\end{tabular}

Notas:

\# Gumbricht et al. (2017)

\& Solo en bofedales, según Chimner (2019) basado en en Chimner et al. (2019)

$\$$ No existen estudios que permitan cuantificar el área de turberas en la zona costera. 
de graminoides (p. ej., Cortaderia spp.) (Chimner et al. 2019), y ciperáceas y musgos (Sphagnum spp.) (Cooper et al. 2010; Benavides y Vitt 2014).
En zonas costeras se ha observado la presencia de turba en manglares (MINAM 2018b) y en juncales (Ampuero y Aponte 2020).

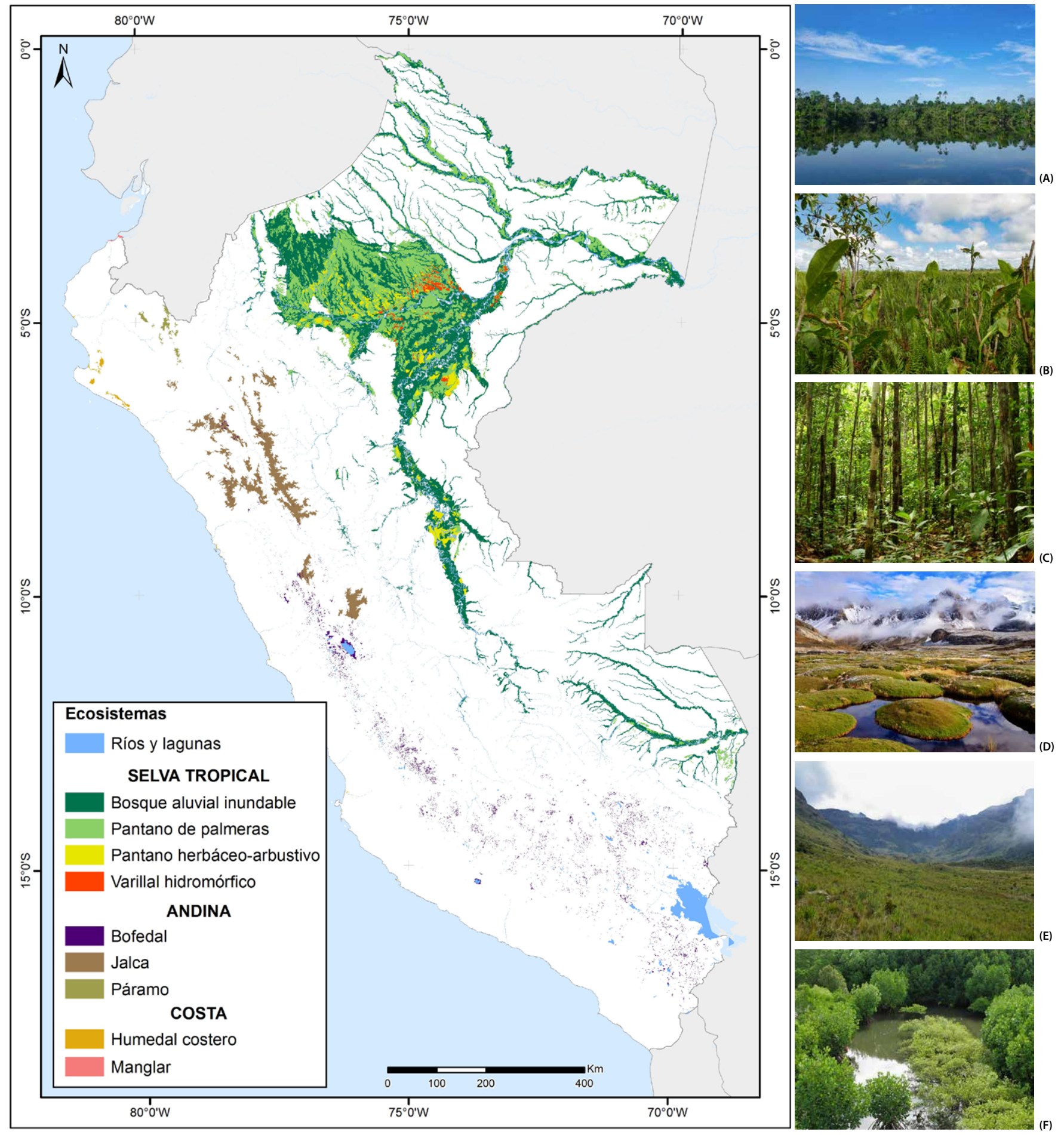

Figura 1. Ecosistemas con potencial de formación de turba en el Perú

Adaptado del mapa de ecosistemas del MINAM (2018b), con datos de varillal hidromórfico del estudio de Draper et al. (2014). (A) Pantano de palmeras (@ Kristell Hergoualc'h), (B) Pantano herbáceo arbustivo (๑ lan T. Lawson), (C) Varillal hidromórfico (๑ lan T. Lawson), (D) Bofedal (๑ Todd Ontl), (E) Páramo (๑ Erik Lilleskov), (F) Manglar (@ Kristell Hergoualc'h). 
Investigaciones indican que la formación de las turberas peruanas se inició hace aproximadamente 8900 años en la Amazonía (Swindles et al. 2017) y 9300 años en las zonas altoandinas (Planas et al. 2020). Los promedios de acumulación de turba reportados van de 1,9 a 2,2 $\mathrm{mm}^{2}$ año ${ }^{-1}$ (Lähteenoja et al. 2009; Roucoux et al. 2013; Swindles et al. 2017), por lo que $10 \mathrm{~cm}$ de turba representarían alrededor de 50 años de acumulación, sin tomar en cuenta la influencia de otros factores como la hidrología y el clima. Dataciones de $\mathrm{C}$ revelaron tasas promedio de acumulación de $66 \mathrm{~g} \mathrm{C} \mathrm{m}^{-2}$ año ${ }^{-1}$ en turberas amazónicas (Lähteenoja et al. 2009) y $59 \mathrm{~g} \mathrm{C} \mathrm{m}^{-2}$ año $^{-1}$ en los Andes (Huamán et al. 2017). Estas tasas de acumulación demuestran el importante rol de secuestro de $\mathrm{C}$ que cumplen las turberas peruanas.

Las propiedades de la turba y de sus ecosistemas asociados están influenciadas fuertemente por la topografía y el clima a largo plazo. Por ejemplo, en el norte de la cuenca del Pastaza-Marańón, la gran estabilidad del río Tigre ha permitido la formación de varillales hidromórficos, en los que la turba es muy profunda y se acumula por encima del nivel de inundación fluvial (Draper et al. 2014). Esto conlleva a su condición ombrotrófica (alimentación solo por precipitaciones), con suelos ácidos y pobres en nutrientes, pero altos en contenido de C. En contraste, las turberas minerotróficas, alimentadas por lluvias y agua de río superficial o subterránea, son más ricas en nutrientes, pero tienen un contenido de $\mathrm{C}$ menor que los sistemas ombrotróficos. Se estima que, en el Perú, el $60 \%$ de las turberas amazónicas son minerotróficas (Lähteenoja y Page 2011), mientras que los datos sobre las turberas andinas son insuficientes para proveer una estimación. Sin embargo, la gran mayoría de las turberas estudiadas en la puna y la jalca son minerotróficas ( $f e n$ en inglés; Cooper $e t a l$. 2010; Chimner et al. 2019). Las turberas de bofedal son comúnmente designadas como ombrotróficas (bog en inglés), aunque sus características hidrológicas sugieren un estado minerotrófico (Cooper et al. 2010; Chimner et al. 2019).

Las turberas peruanas constituyen reservorios enormes de C. Solo en la cuenca del PastazaMarañón, que alberga el $3 \%$ del área boscosa del país (Saatchi et al. 2007), se ha estimado la presencia de 3,1 Gt C en el suelo y la vegetación de las turberas (Draper et al. 2014). Estas reservas representan alrededor del $50 \%$ del C almacenado en la biomasa aérea en todo el Perú (6,9 Gt C; Asner et al. 2014) o el equivalente de más de 90 años de sus emisiones antropogénicas de $\mathrm{CO}_{2}\left(0,13 \mathrm{Gt} \mathrm{CO}_{2}\right.$ en 2014; MINAM 2019). En la Amazonía, la reserva total (suelo y vegetación) de $\mathrm{C}$ por unidad de área es, en promedio, de 2 a 4 veces mayor en turberas que en bosques de tierra firme (Baker et al., 2020). Esta diferencia se debe principalmente a las inmensas reservas encontradas en el suelo. En la cuenca del Pastaza, se registraron profundidades de turba de hasta 7,5 m (Lähteenoja et al. 2012). En pantanos de palmeras, Bhomia et al. (2019) midieron almacenamientos de $\mathrm{C}$ total dentro de un rango de 200-1600 t C ha-1, con el -75\% (109-594 t C ha-1) concentrado en los primeros $100 \mathrm{~cm}$ del suelo. Los datos de profundidad o de almacenamiento de $\mathrm{C}$ en turberas altoandinas son más escasos, pero se encontraron bofedales con una profundidad de turba de hasta $10,5 \mathrm{~m}$ y una reserva de $2100 \mathrm{t} \mathrm{C} \mathrm{ha}^{-1}$ en el suelo (Hribljan et al. sin publicar). Asimismo, de las 36 parcelas en humedales de jalca muestreadas por Cooper et al. (2010) en Cajamarca, el $17 \%$, más del $25 \%$ y más del $50 \%$ tenían, respectivamente, una profundidad de turba mayor de $7 \mathrm{~m}, 5 \mathrm{~m}$ y $3 \mathrm{~m}$.

Además de su mayor contribución en el almacenamiento y secuestro de $\mathrm{C}$, las turberas cumplen un papel fundamental en la regulación del ciclo hidrológico mediante el paisaje y proporcionan un suministro constante de agua durante la época de estiaje (caudal base) (Maldonado Fonkén 2014). Este efecto de regulación hidrológica hace que las regiones donde se encuentran las turberas sean más resilientes a efectos adversos del cambio climático como sequías e inundaciones prolongadas (García y Otto 2015).

Por otro lado, las turberas son el hogar de una biodiversidad única. Una gran variedad de animales vive en los pantanos de palmeras o los recorren para obtener alimentos y agua (Gilmore, Endress y Horn 2013). Entre ellos, monos, guacamayos (en especial el guacamayo azul y amarillo [Ara ararauna] y el guacamayo de vientre rojo [Orthopsittaca manilatus]), ungulados (en especial el tapir [Tapirus terrestris] y el pecarí labiado [Tayassu pecari]), roedores (como el agutí [Dasyprocta spp.] y la paca [Agouti paca]), tortugas (Chelonoidis denticulata) y peces. El fruto de $M$. flexuosa, en particular, cumple un papel muy importante en la ecología y el ciclo de vida de numerosas especies, en especial de los ungulados antes mencionados (Bodmer $e t$ al. 1999). Algunas especies de aves están prácticamente especializadas en los pantanos de $M$. flexuosa, como el trepador de palmeras (Berlepschia rikeri), el bolsero moriche (Icterus chrysocephalus) y el mosquero 
azufrado (Tyrannopsis sulphurea). Los varillales hidromórficos también son el hábitat de aves amenazadas y en peligro de extinción y mantienen especies asociadas con otros hábitats. Por su parte, los bofedales albergan una gran variedad de camélidos y roedores, y sirven como espacio para la alimentación y nidificación de aves, entre ellas, especies amenazadas como el churrete de vientre blanco (Cinclodes palliatus) (Maldonado Fonkén 2014).

Tanto las turberas de las tierras bajas como de las zonas altoandinas constituyen medios de subsistencia fundamentales para las comunidades locales. En la Amazonía, las comunidades nativas y ribereńas utilizan los recursos de los ecosistemas de turberas para la pesca, la caza de animales y la recolección de recursos vegetales. Más de 50 especies de plantas son aprovechadas para distintos fines y forman parte de la economía local, entre las que destaca el fruto de $M$. flexuosa de los pantanos de palmeras, cuyo consumo alcanza las 685 toneladas por mes solo en la ciudad de Iquitos (Horn et al. 2018). En zonas de bofedales donde las condiciones climáticas hacen inviable la agricultura, la ganadería es la principal fuente de ingresos económicos (Maldonado Fonkén, 2014). El ganado se alimenta principalmente de la vegetación herbácea, por lo que la humedad del subsuelo encontrada en las turberas es indispensable para asegurar la producción de forraje de calidad y, con ello, la disponibilidad de carne y fibras, en especial en las partes más secas de los Andes y durante los meses más secos del año. Los bofedales son también muy importantes para la alimentación de la fauna silvestre, entre la que destaca la vicuña (Vicuana vicuana), cuya fibra es una fuente de ingresos importante para las comunidades rurales.

Por último, las turberas son parte fundamental de las raíces culturales de muchas comunidades indígenas, cuyos miembros tienen una cosmovisión que integra aspectos físicos y espirituales, poseen un conocimiento detallado de su entorno y son conscientes de la importancia y el manejo sostenible de los humedales (Martín et al. 2019; Schulz et al. 2019). Tal es el caso del pueblo urarina en Loreto y del pueblo achuar del Pastaza, cuyo nombre proviene de la palmera de $M$. flexuosa (que en achuar se denomina $a c h u$ ), a la que sus miembros consideran sagrada (Kahn et al. 1993). Asimismo, dentro de la cosmovisión andina, los humedales son conocidos por las comunidades campesinas como oconales, nombre derivado de la palabra quechua oqho, que hace referencia a los pastos naturales en ambientes húmedos (Salvador y Cano 2002). Las comunidades valoran estos ecosistemas no solo porque aprovechan sus recursos (sobre todo para mantener al ganado) sino también por su importancia cultural, que genera un respeto al ecosistema y beneficia su conservación. Cabe resaltar también que muchos humedales altoandinos, incluidas las turberas, son artificiales y han sido gestionados por la población andina por más de 2000 ańos mediante la creación de canales para el manejo del agua y para aumentar su extensión (Verzijl y Guerrero 2013).

\subsection{Amenazas}

La tabla 2 resume las causas de la degradación de las turberas comunes a todas las regiones del Perú y específicas de cada región. Cualquiera de estas formas de degradación trae consigo pérdidas cruciales de $\mathrm{C}$ y emisiones de GEI en proporciones muy superiores a las de ecosistemas de suelos minerales (Drösler et al. 2014; Oktarita et al. 2017).

En la Amazonía, alrededor del $24 \%$ de las turberas de la cuenca del Pastaza se encuentran en concesiones petroleras (Roucoux et al. 2017). Ello, junto con el desarrollo de redes viales y otras infraestructuras proyectadas, podría alterar la conectividad hidrológica de la cuenca y potenciar una migración poblacional que resulte en la aceleración de la degradación de las turberas debido

Tabla 2. Principales causas de la degradación de las turberas en el Perú

\begin{tabular}{ll}
\hline Región & Causas de la degradación \\
\hline Todas & $\begin{array}{l}\text { Actividades extractivas, productivas y de servicios de alto impacto, como la explotación de } \\
\text { recursos petroleros y mineros y la construcción de vías; cambios climáticos. }\end{array}$ \\
Amazonía & Tala selectiva de palmeras, cambio de uso de la tierra, sobrecaza. \\
Andes & Sobrepastoreo, drenaje, extracción de turba. \\
Costa & Incendios antropogénicos. \\
\hline
\end{tabular}


a la ampliación de la frontera agrícola, la minería aurífera y la sobreexplotación de recursos (Roucoux et al. 2017; Lilleskov et al. 2017). Por ejemplo, se proyecta construir una carretera que conecte la región Loreto con la costa norte del Perú (carretera Iquitos-Saramiriza; Ley N.o 30670, República del Perú 2017). Aunque el trazado de la carretera en sí se ubica más allá del límite del complejo de humedales del Abanico del Pastaza, el sitio Ramsar más grande de la Amazonía peruana, pasaría cerca de los depósitos de turba más profundos de toda la cuenca (Baker et al. 2020). Además, hace más de 5 años se anunció el proyecto de la línea de transmisión eléctrica que conectaría a Iquitos con el Sistema Eléctrico Interconectado Nacional (SEIN), uno de cuyos tramos, según el proyecto del Ministerio de Energía y Minas, consideraba atravesar el citado Abanico del Pastaza en su sección más ancha.

Los ecosistemas de turba que han sufrido mayor degradación en las últimas décadas son los pantanos de palmeras o aguajales. Desde hace más de 40 años (Padoch 1988), han sido explotados de una manera no sostenible al cosechar los frutos de $M$. flexuosa mediante la tala selectiva de palmeras hembras (Horn et al. 2012). Un estudio piloto estimó que, en un área de 350000 hectáreas en la cuenca de los ríos Ucayali y Marañón, el $73 \%$ de los aguajales se encontraban degradados (Hergoualc'h et al. 2017). Con el tiempo, esta práctica reduce los recursos, modifica la estructura y composición del bosque, estimula la descomposición de la turba y, potencialmente, suspende su formación (Hergoualc'h et al. 2017; van Lent et al. 2019). El impacto de esta práctica en las emisiones de óxido nitroso y de metano de la turba se concentra en la microescala y no tanto a nivel del ecosistema (Hergoualc'h et al. 2020). Si bien la agricultura comercial aún no se ha expandido ampliamente en las turberas amazónicas, se han reportado casos de establecimiento de plantaciones y arrozales en humedales y pantanos de palmeras en regiones como Madre de Dios (Janovec et al. 2013), Ucayali (comunicación personal de I Núñez, 2020) y San Martín (Noriega 2012). Por último, la sobrecaza, tal como fue observada en el norte de la Amazonía peruana (Aquino et al. 2007), disminuye las poblaciones animales, lo que puede reducir la dispersión de semillas y alterar la composición y estructura de los ecosistemas y afectar sus funciones, como el almacenamiento de C (Bagchi et al. 2018; Peres et al. 2016).
En los Andes, la actividad minera y la construcción de carreteras y represas alteran la hidrología y/o contaminan suelos y aguas con sedimentos y sustancias tóxicas (Maldonado Fonkén 2014). Esto ocasiona daños a gran escala, inmediatos y a largo plazo, que repercuten en las poblaciones aledañas y aguas abajo. Los bofedales han sido zonas de pastoreo desde épocas prehispánicas. No obstante, el creciente sobrepastoreo (en especial por animales de pezuña introducidos), la quema, el mal manejo y la construcción de zanjas están ocasionando su pérdida progresiva (Román-Cuesta et al. 2011; Salvador, Monerris y Rochefort 2014; García y Otto 2015; ANA 2018; Planas et al. 2020). El sobrepastoreo provoca cuatro tipos de impacto principales: (a) disminuye el aporte de materia orgánica al suelo, lo que interrumpe la formación de turba; (b) modifica las propiedades físicas del suelo (p. ej., compactación); (c) aumenta los flujos de metano (Sánchez et al. 2017); y (d) propicia la descomposición de la turba en casos de drenaje para facilitar el acceso del ganado (Planas et al. 2020). La falta de delimitación y zonificación de las turberas en zonas de bofedales obstaculiza los esfuerzos para un mejor manejo que redirija el pastoreo hacia áreas sin turba (Chimner et al. 2019). Por otro lado, la extracción de turba para su uso en cultivos de agroexportación y horticultura, realizada por personas externas a las comunidades, ha aumentado de manera alarmante en los últimos años (Machuca 2018; Ziegler 2020), lo cual afecta el sistema de producción pecuaria y contribuye al agotamiento rápido y la pérdida irreparable del recurso.

En la costa, los incendios antropogénicos en humedales ocurren con alta frecuencia (Aponte et al. 2015), lo que produce consecuencias aún no evaluadas en la diversidad biológica, libera altas cantidades de GEI a la atmósfera y reduce sustancialmente las reservas de $\mathrm{C}$ (Ampuero y Aponte 2020).

Las turberas también están amenazadas por los cambios climáticos globales y pueden ser fuertemente afectadas por la intensificación de eventos climáticos como sequías o inundaciones y el aumento de la temperatura (Roucoux et al. 2017; Swindles et al. 2017). Según Swindles et al. (2017), sequías futuras en la Amazonía tendrían consecuencias diversas dependiendo del ecosistema. Podrían propiciar fases de acumulación rápida de $\mathrm{C}$ en algunos pantanos de turba (por aumentar su productividad primaria neta), lo que en última instancia podría conducir a una ombrotroficación 
del sistema (es decir, la sucesión de un estado minerotrófico a un estado ombrotrófico) y un posterior retorno a una acumulación más lenta de C. En cambio, en domos de turba ombrotróficos, las sequías podrían reducir la acumulación de $\mathrm{C}$ o incluso inducir a una pérdida neta de turba. Periodos de sequía de mayor duración, acompañados por una reducción de la capa freática, también favorecerían los incendios (Lilleskov et al. 2017). Un clima más húmedo (Swindles et al. 2017) e inundaciones fuertes, como las que ocurrieron en las últimas décadas en la zona oeste de la Amazonía (Gloor et al. 2013), tendrían impactos sobre el secuestro de $\mathrm{C}$ potencialmente opuestos a los antes descritos, inducidos por las sequías. Un incremento de las precipitaciones también estimularía las emisiones de metano en pantanos de palmeras (Hergoualc'h et al. 2020). En los Andes, aunque la vulnerabilidad de las turberas al cambio climático está vinculada estrechamente a una menor disponibilidad de agua (ANA 2018), el retroceso de los glaciares encubre procesos que van más allá de la afectación de sus reservas hídricas. Entre ellos está la erosión extrema que sufren áreas que se han derretido y que contienen sustancias tóxicas, como el drenaje ácido de roca, que contamina los bofedales y afecta a las poblaciones locales y su ganadería (Angulo y De Biévre 2017; INAIGEM 2017). Por otro lado, un aumento de temperatura de $0,5^{\circ} \mathrm{C}$ para el año 2100 (escenario RCP 2.6 del IPCC, el Panel Intergubernamental del Cambio Climático; IPCC 2014) en la región del PastazaMarañón disminuiría la tasa de acumulación de C orgánico de la turba (Wang et al. 2018). Un aumento de $2,7^{\circ} \mathrm{C}$ (escenario RCP 8.5) aceleraría su pérdida de $\mathrm{C}$, lo que convertiría a las turberas de la región en una fuente de $\mathrm{C}$ lanzado a la atmósfera a razón de $53 \mathrm{~g} \mathrm{C} \mathrm{m}^{-2}$ año ${ }^{1}$.

\subsection{Marco normativo}

En el ámbito nacional, se cuenta con normas e instrumentos vinculados a la gestión sostenible de los humedales, que incluyen a las turberas. Pero, aunque el marco normativo incluye a los humedales y hace referencia a los pantanos de palmeras, a los bofedales y a las turberas, no desarrolla una definición ni regulaciones específicas para las turberas, lo que pone en riesgo su integridad. Dicha inclusión es necesaria para velar por su gestión sostenible de la mano de la evidencia científica existente, dada su importancia, vulnerabilidad y relevancia para el cumplimiento de los compromisos internacionales del Perú en el marco del Acuerdo de París y otros acuerdos internacionales. Por ejemplo, la Estrategia Nacional de Humedales (MINAM 2015), cuyo objetivo es promover la conservación y el uso sostenible de los humedales, requeriría considerar la gran variedad de turberas existente en el país, su extensión diferenciada de otros humedales y su papel clave en el almacenamiento y secuestro de C. Por otro lado, dado que la gran mayoría de las turberas del país son bosques, se requiere que la Estrategia Nacional sobre Bosques y Cambio Climático (ENBCC; MINAM 2016) mencione la Estrategia Nacional de Humedales. De igual manera, es de suma importancia que la ENBCC, en su análisis de las causas subyacentes de la deforestación, suprima la recomendación de "Brindar asesoramiento para implementar o mejorar algunas tecnologías, como las que se usan para drenar los humedales" para "reducir la migración de la población indígena y campesina hacia suelos fértiles y/o zonas no deforestadas" (MINAM 2016, 60). Si bien el país está comprometido a hacer frente al cambio climático y ha formulado metas de adaptación y mitigación en sus Contribuciones Nacionalmente Determinadas (NDC, por sus siglas en inglés) como parte del Acuerdo de París, aún no contempla el potencial de las turberas en sus medidas de mitigación de emisiones de GEI o de adaptación al cambio climático (República del Perú 2015). La actualización de las NDC considera los humedales y los bofedales en cuatro medidas relativas a la adaptación al cambio climático, pero está pendiente la inclusión explícita de las turberas, en especial en las medidas de mitigación (GTM-NDC 2018). Asimismo, falta aún incorporar las turberas y sus inmensas reservas de $\mathrm{C}$ en la reducción de emisiones derivadas de la deforestación y la degradación de los bosques (REDD+) del Perú.

Ciertas zonas de turberas (p. ej., la Reserva Nacional Pacaya Samiria, Tamshiyacu Tahuayo, la Sierra del Divisor, etc.) se encuentran protegidas por estar incluidas en zonas forestales de conservación y protección ecológica y en áreas naturales protegidas (Ley N.o 26834, República del Perú 1997). Del mismo modo, existen parques nacionales, como el recientemente creado Parque Nacional Yaguas (Decreto Supremo N.o 001-2018-MINAM, MINAM 2018a), que albergan extensas áreas de pantanos de turba (Baker et al. 2020). Sin embargo, en la cuenca del Pastaza-Marañón, la gran mayoría de las turberas, 
aunque forman parte de un sitio Ramsar y en cierta extensión están incluidas en territorios titulados a comunidades indígenas, requieren de instrumentos complementarios para su protección y gestión efectiva. Por ejemplo, los varillales hidromórficos del río Tigre - los ecosistemas de turbera con la más alta densidad de $\mathrm{C}$ medida hasta el momento en la cuenca amazónica- solo cuentan con denominación de sitio Ramsar (Roucoux et al. 2017). De la misma manera, extensas zonas de bofedales altoandinos no tienen ningún tipo de protección, aun cuando representan invaluables zonas de captación, almacenamiento y regulación de agua, que es esencial para la población circundante y en las zonas bajas de estas cuencas.

Por otro lado, el país cuenta con importantes regulaciones vinculadas a mecanismos de pago por servicios ecosistémicos. A nivel nacional, la SUNASS (Superintendencia Nacional de Servicios de Saneamiento) viene incorporando en las tarifas de agua de diversas EPS (Empresas Prestadoras de Servicios de Saneamiento) provisiones para proyectos de conservación y/o recuperación de ecosistemas de interés hídrico, en los que se incluyen ecosistemas con potencial de formación de turba (Ley N.o 30215, Decreto Supremo N. ${ }^{\circ}$ 009-2016-MINAM). De igual manera, en el marco del esquema de pago por servicios ecosistémicos, el proyecto MERESE-FIDA canaliza recursos para acciones de conservación, recuperación y uso sostenible de ecosistemas en zonas altoandinas, incluidos bofedales, mediante un financiamiento del Fondo para el Medioambiente Mundial (FMAM, o GEF por sus siglas en inglés) y bajo la administración del MINAM (Ley N. ${ }^{\circ} 30215$, República del Perú 2016).

Por último, el Perú cuenta con instancias de coordinación interinstitucional vinculadas a los humedales, como es el caso del Comité Nacional de Humedales $(\mathrm{CNH})$, orientado a promover la gestión adecuada de los humedales a nivel nacional, así como a hacer seguimiento a la implementación de los compromisos derivados de la Convención de Ramsar. 


\section{Oportunidades y retos}

Para la conservación y el uso sostenible de las turberas en el Perú, se requiere fortalecer el marco normativo, los mecanismos financieros y de promoción de la inversión pública y privada (incluidas las comunidades locales), la investigación científica y la revaloración de los conocimientos indígenas.

Lilleskov et al. (2017) hicieron una lista de las opciones de políticas que necesitan incluir de manera explícita a las turberas. Con base en esta lista, se sugiere evaluar, promover o desarrollar aquellas relacionadas con: (a) la prohibición del drenaje de turberas para cualquier tipo de actividad, y del desarrollo de vías de circulación o exploración y de infraestructura para la extracción de recursos (minerales, gas, petróleo) en estos ámbitos; (b) un planeamiento agrícola y forestal que incluya las vulnerabilidades únicas de las turberas (p. ej., ante los riesgos de incendios); y (c) la implementación efectiva de regulaciones para aquellas actividades que afectan a las turberas, como, por ejemplo, la tala ilegal o la extracción y comercialización de turba.

Con el fin de impulsar una conservación efectiva de las turberas, es esencial fortalecer la coordinación multisectorial e interinstitucional entre los diversos actores y niveles de gobierno. Se requiere precisar las funciones de las diversas instituciones involucradas en su conservación y gestión: MINAM, MINAGRI (Ministerio de Agricultura y Riego) - y sus adscritos SERFOR (Servicio Nacional Forestal y de Fauna Silvestre) y ANA (Autoridad Nacional del Agua)_, PRODUCE (Ministerio de la Producción), gobiernos subnacionales y otros actores clave. El CNH es la instancia de articulación adecuada en este sentido y un espacio apropiado para promover el debate para la mejora de la gestión integral de las turberas. Recientemente, el CNH ha empezado a tomar medidas para promover la gestión sostenible de las turberas. Entre las medidas iniciales, destaca la elaboración de una guía técnica para la definición de las turberas en el país, incluida la identificación de ecosistemas con presencia comprobada o con potencial de formar turba. La adopción de una definición nacional consensuada entre los diferentes actores será indispensable para mejorar las políticas existentes o desarrollar nuevas políticas que protejan las turberas al incluirlas en estrategias de mitigación y adaptación al cambio climático, de conservación de la biodiversidad y manejo sostenible de los humedales y de los recursos hídricos, y de lucha contra la desertificación y la sequía. También será de gran utilidad para destacar la importancia de las turberas como reguladores hídricos en infraestructuras verdes, como parte de los Objetivos de Desarrollo Sostenible (ODS) asumidos por el país, de manera específica, en relación con la meta sobre agua y saneamiento (ODS 6). Por otro lado, la ANA, en coordinación con el CNH, está generando información con un enfoque hidrológico para contribuir al desarrollo del inventario nacional de humedales, instrumento técnico que busca coadyuvar a la gestión y conservación de los humedales en los diferentes niveles de gobierno (nacional, regional y local). Dicho inventario sentará las bases para la identificación y caracterización de las turberas en el país. Por su parte, el MINAM también está impulsando la aprobación de una norma nacional para promover la conservación y gestión integral de los humedales en el territorio nacional, entre los que se incluyen las turberas.

Es importante que la actualización de las NDC y del mecanismo REDD+ considere de manera explícita las turberas. Aunque el país todavía no cuenta con datos de actividades y factores de emisiones precisos, existe información suficiente para fijar objetivos de reducción de emisiones de las turberas. Por ejemplo, la implementación efectiva de un plan de manejo sostenible de pantanos de palmeras turbosos, que evite la tala destructiva de las palmeras mediante la cosecha de frutos con subidores y rehabilite un área muy degradada de $277 \mathrm{~km}^{2}$ (solo el $1 \%$ de la superficie de este ecosistema en el Pastaza-Marañón, según Draper et al. 2014), permitiría una reducción 
de las emisiones de $\mathrm{CO}_{2}$ del suelo de 0,54 $\mathrm{Mt}_{\text {año }}{ }^{-1}$ $\left(1 \mathrm{Mt}=10^{12} \mathrm{~g}\right)$. Estas estimaciones, proyectadas sobre un periodo de 10 años, representan hasta el $6 \%$ de las metas de reducción actuales del país para el año $2030\left(89 \mathrm{Mt} \mathrm{CO}_{2 \mathrm{eq}}\right)$. Están basadas en el factor de emisión de $\mathrm{CO}_{2}$ del IPCC para la descomposición de la turba en bosques drenados (5,3 t C ha ${ }^{-1}$ año $^{-1}$; tabla 2.1 en Drösler $e$ t al. 2014) y subestiman el potencial de mitigación, dado que excluyen el secuestro de $\mathrm{C}$ mediante la regeneración de la vegetación.

Una oportunidad mayor de conservación de las turberas peruanas se presenta mediante el Sistema Nacional de Áreas Naturales Protegidas por el Estado (SINANPE). Cerca de la tercera parte del total de ANP en el Perú tendría bofedales (Maldonado 2010), aunque se desconoce la cobertura específica. En la Amazonía, el 23 \% del complejo de turberas del Pastaza-Marañón está ubicado en grandes reservas nacionales $(\mathrm{RN})$ y regionales como la RN Pacaya Samiria (Roucoux et al. 2017). Se ha demostrado que en ANP se puede promover un manejo sostenible de los recursos de flora y fauna silvestre, así como de los hidrobiológicos, mediante el desarrollo de ciertas actividades extractivas de recursos naturales renovables por las comunidades. Tal es el caso de la RN Pacaya Samiria, donde el programa para la cosecha sostenible de frutos mediante la técnica de escalamiento de palmeras de aguaje genera importantes ingresos a los pobladores locales y evita la degradación del bosque al dar valor agregado a los recursos (Falen y Honorio 2018; SERNANP 2020). La ampliación de ANP a zonas de turberas debe tomar en cuenta la vulnerabilidad del ecosistema a la degradación provocada por el ser humano y los servicios ecosistémicos que este ofrece como sumidero y depósito de C, así como su potencial de conservación de agua y de control de inundaciones. La conservación mediante el manejo sostenible de las turberas que se encuentran fuera de las ANP también puede realizarse priorizándolas como zonas de conservación y protección ecológica en el proceso de zonificación forestal del país y los procesos de zonificación ecológica y económica (ZEE) que impulsan los gobiernos subnacionales. La tenencia de tierras por comunidades campesinas e indígenas es reconocida como otro factor clave para la gestión y conservación de ecosistemas, además de que contribuye a reducir la pobreza y la inseguridad alimentaria (MINAM 2015). Las comunidades poseen actualmente solo el $7 \%$ del área total de turberas en la cuenca del Pastaza-
Marañón (Roucoux et al. 2017), porción que es aún muy insuficiente para su conservación, lo que debe ser tomado en cuenta en los procesos de planificación para el desarrollo de nuevas obras de infraestructura y otras actividades que pudieran generar impactos. Las evidencias que conectan la pérdida de los conocimientos tradicionales con la degradación de los ecosistemas son cada vez más numerosas (Harmon 1996; Ross 2002; Loh y Harmon 2014; Pröpper y Haupts 2014; Schulz et al. 2019). Es por ello imprescindible preservar los conocimientos, los valores y las prácticas tradicionales de los pueblos indígenas, y fortalecer las capacidades adaptativas de las comunidades para responder a nuevas presiones y cambios, lo que sin duda contribuirá a conservar las turberas en el Perú (Martín et al. 2019). Cualquiera sea la opción (u opciones) que se elija para la conservación y el manejo sostenible de turberas, se debe considerar la equidad de la estrategia y su eficiencia en costos, así como los riesgos asociados de conflictos y mala gobernanza.

La conservación, gestión sostenible, recuperación y puesta en valor de las turberas necesitan inversiones públicas y privadas, nacionales e internacionales. Entre las iniciativas en curso, el proyecto Humedales del Datem del Marañón ejecutado por PROFONANPE (Fondo Nacional para Áreas Naturales Protegidas por el Estado) con financiamiento del Fondo Verde para el Clima, integra iniciativas de protección de las reservas de $\mathrm{C}$ en aguajales y de resiliencia de las poblaciones indígenas mediante la promoción de bionegocios. Otra de estas iniciativas es el proyecto Infraestructura Natural para la Seguridad Hídrica financiado por USAID (Agencia de los Estados Unidos para el Desarrollo Internacional), que promueve la conservación, restauración y recuperación de los ecosistemas, en especial de los bofedales, para la seguridad hídrica en el país. Entre las inversiones del sector privado está el proyecto de bebidas BIO del Grupo AJE en asociación con la ONG Naturaleza y Cultura Internacional, la cual brinda asistencia técnica en la organización, entrenamiento y equipamiento de las comunidades para cosechar los frutos de $M$. flexuosa sin cortar la palmera ni degradar el bosque (Tropical Forest Alliance 2020).

Tanto el fortalecimiento del marco normativo como el de las actividades de conservación y gestión de los ecosistemas de turba deben basarse en información científica sólida. A pesar de que existen 
guías, herramientas o estudios globales — como el mapa global de humedales (Gumbricht et al. 2017) o el suplemento de 2013 del IPCC para inventarios nacionales de GEI en humedales (Hiraishi et al. 2014) — que ya permiten proceder con la preparación de inventarios preliminares, es necesario que el país desarrolle sus datos propios. En especial es importante que, como primer paso, el Perú cuente con un mapa y un inventario nacional de sus turberas, que faciliten su localización y cuantifiquen su extensión y reservas de $\mathrm{C}$, que actualmente se evalúan de manera aproximada mediante estudios específicos. Existen aún importantes vacíos de investigación relativos a las turberas peruanas, a pesar de los avances significativos de los últimos años (Draper et al. 2014; Chimner et al. 2019; Van Lent et al. 2019; Hergoualc'h et al. 2017; Bhomia et al. 2019; Freitas et al. 2019; Falen y Honorio 2018; Schulz et al. 2019, Griffis et al. 2020). La tabla 3 presenta áreas de investigación necesaria para profundizar en conocimientos esenciales para la protección y el manejo sostenible de las turberas del país.

Tabla 3. Objetivos y necesidades de investigación científica para la protección y el manejo sostenible de las turberas peruanas

\begin{tabular}{|c|c|}
\hline Objetivos & Necesidades de investigación \\
\hline \multirow{4}{*}{$\begin{array}{l}\text { Conocimiento de } \\
\text { la distribución, el } \\
\text { funcionamiento y los } \\
\text { servicios ecosistémicos de } \\
\text { las turberas }\end{array}$} & Clasificación y mapeo nacional de los ecosistemas de turba y de sus reservas de $C$. \\
\hline & $\begin{array}{l}\text { Medición detallada del almacenamiento de } C \text { en ecosistemas de turba, con } \\
\text { énfasis en el } C \text { del suelo e inventarios de su biodiversidad. }\end{array}$ \\
\hline & Monitoreo a largo plazo de balances hídricos, de materia orgánica y de flujos de GEl. \\
\hline & $\begin{array}{l}\text { Estudios hidrogeológicos en turberas y modelamiento de las necesidades de } \\
\text { agua: caudal ecológico en zonas con déficit hídrico y cabeceras de cuencas. }\end{array}$ \\
\hline \multirow{5}{*}{$\begin{array}{l}\text { Conocimiento de los } \\
\text { agentes de la degradación } \\
\text { de las turberas, y de los } \\
\text { impactos, la extensión } \\
\text { y la localización de la } \\
\text { degradación }\end{array}$} & $\begin{array}{l}\text { Identificación de los agentes de degradación (p. ej., obras de infraestructura, } \\
\text { minería, sobrepastoreo en zonas altoandinas, tala de } M \text {. flexuosa en la Amazonía, } \\
\text { cambios en el uso del suelo, contaminación, etc.) y su localización geográfica. }\end{array}$ \\
\hline & $\begin{array}{l}\text { Mapeo de los usuarios que identifique la base jurídica o consuetudinaria de sus } \\
\text { derechos y usos. }\end{array}$ \\
\hline & $\begin{array}{l}\text { Mapeo y monitoreo de la degradación de las turberas según niveles de } \\
\text { intensidad. }\end{array}$ \\
\hline & $\begin{array}{l}\text { Evaluación del efecto de la degradación en las reservas y el secuestro de C, los } \\
\text { flujos de GEl, los flujos hídricos y los recursos (p. ej., forraje en zonas altoandinas, } \\
\text { frutos de M. flexuosa en la Amazonía). }\end{array}$ \\
\hline & $\begin{array}{l}\text { Evaluación del impacto del cambio climático en el funcionamiento de las } \\
\text { turberas, como su capacidad de secuestro de } C \text {, de regulación hídrica o de } \\
\text { provisión de recursos (p. ej., modificaciones en el comportamiento de la fenología } \\
\text { reproductiva y de la producción de frutos de M. flexuosa en la Amazonía). }\end{array}$ \\
\hline \multirow{3}{*}{$\begin{array}{l}\text { Conocimiento para } \\
\text { reducir las presiones } \\
\text { antropogénicas sobre los } \\
\text { ecosistemas de turberas }\end{array}$} & $\begin{array}{l}\text { Investigación y revalorización de los conocimientos y prácticas tradicionales de } \\
\text { las comunidades campesinas e indígenas. }\end{array}$ \\
\hline & $\begin{array}{l}\text { Estudio del manejo sostenible de bosques naturales, técnicas de forestería y } \\
\text { plantaciones (p. ej., mejoramiento genético de la especie } M \text {. flexuosa para su } \\
\text { establecimiento en plantaciones). }\end{array}$ \\
\hline & $\begin{array}{l}\text { Estudios de aprovechamiento de los recursos del ecosistema para un uso } \\
\text { más integral (p. ej., valor nutricional de partes florales y polen de la especie } \\
\text { M. flexuosa). }\end{array}$ \\
\hline \multirow{3}{*}{$\begin{array}{l}\text { Conocimiento para la } \\
\text { recuperación de turberas } \\
\text { degradadas }\end{array}$} & Evaluación de la localización y la intensidad de las necesidades de restauración. \\
\hline & $\begin{array}{l}\text { Estudio de técnicas de restauración de turberas, como la recuperación del nivel } \\
\text { freático o la plantación de } M \text {. flexuosa en pantanos de palmeras degradados. }\end{array}$ \\
\hline & $\begin{array}{l}\text { Evaluación de la efectividad de tratamientos de restauración en las condiciones } \\
\text { hidrológicas, el secuestro de C, las emisiones de GEl, etc. }\end{array}$ \\
\hline
\end{tabular}




\section{Recomendaciones}

Para mantener la integridad de las turberas peruanas es necesario que estén identificadas, mapeadas y reconocidas de manera explícita por las políticas gubernamentales. Como primer paso, se requiere adoptar una definición nacional de "turbera", lo que permitirá su inclusión en normativas existentes o el desarrollo de nuevas normativas.

El $\mathrm{CNH}$, que coordina de manera multisectorial e interinstitucional los aspectos relacionados con la Convención de Ramsar, es el espacio adecuado para liderar un fortalecimiento del marco legal vigente que otorgue un carácter prioritario a las turberas. Es esencial que el CNH colabore estrechamente con los gobiernos regionales y locales, los organismos públicos pertinentes y otros actores claves en la gestión de las turberas, como las organizaciones indígenas y campesinas.

Los programas - públicos o privados- de conservación, manejo sostenible y restauración de turberas deben considerar los objetivos de desarrollo de las comunidades aledañas, con el fin de optimizar los cobeneficios ecológicos, económicos y sociales. Contar con comunidades locales que se beneficien directamente de los bienes y servicios derivados de ecosistemas de turba es la mejor garantía de que estos serán conservados de manera íntegra y funcional. También es necesario que (a) se planteen estrategias de desarrollo de mercados, (b) se invierta en tecnología de transformación para dar valor agregado a los productos de ecosistemas de turba bajo manejo, y (c) se transfieran esas tecnologías a las poblaciones locales. En este aspecto, y dadas las capacidades limitadas de las comunidades, es sumamente importante la participación de empresas privadas con mercado nacional e internacional.

Es necesario fortalecer los derechos de tenencia de las comunidades que habitan en los ecosistemas de turba para que inviertan en buenas prácticas de manejo. También es importante que se valide la sostenibilidad de las prácticas tradicionales en colaboración con las instituciones locales que facilitan la conservación de estas áreas.

Las políticas e intervenciones en turberas deben basarse en evidencia científica. Es imperativo invertir en la profundización y la promoción de los conocimientos científicos, y facilitar su transferencia mediante convenios entre universidades, organismos no gubernamentales, sectores involucrados en la conservación de las turberas y plataformas internacionales (p. ej., GPI, ITPC). También es necesario que se desarrollen programas de capacitación técnica y de educación y divulgación sobre turberas para el público. Por último, es esencial tomar en consideración los conocimientos ancestrales de las comunidades indígenas, lo que permitirá identificar y revalorar antiguas costumbres y técnicas puestas en práctica por las comunidades para la gestión de sus humedales. 


\section{Referencias}

Ampuero W y Aponte H. 2020. Estimación del carbono almacenado en un juncal de Schoenoplectus americanus en el humedal costero Los Pantanos de Villa (Lima-Perú). Arnaldoa 27(1):e157-64.

[ANA] Autoridad Nacional del Agua. 2018. Estudio piloto. Inventario de humedales en el ámbito de la ALA Pisco. ANA.

Angulo Ó y De Biévre B. 2017. Impacts of glaciers retreat on highland Andean wetlands and communities: Lessons from the upper Cachi catchment (Ayacucho, Peru). Geophysical Research Abstracts 19, EGU2017.

Aponte H, Ramírez D, Lértora G, Vargas R, Gil F, Carazas N y Livial R. 2015. Incendios en los humedales de la costa central del Perú: una amenaza frecuente. Cientifica 12(1):70-81.

Aquino R, Terrones C, Navarro R y Terrones W. 2007. Evaluación del impacto de la caza en mamíferos de la cuenca del río Alto Itaya, Amazonía peruana. Revista Peruana de Biología 14(2):181-6.

Asner G, Knapp D, Martin R, Tupayachi R, Anderson C, Mascaro J, Sinca F, Chadwick D, Sousan S, Higgins M, Farfán W, Silman M, Llactayo W y Neyra A. 2014. The highresolution carbon geography of Peru. Carnegie Airborne Observatory and the Ministry of Environment of Peru.

Avitabile V, Herold M, Heuvelink G, Lewis S, et al. 2015. An integrated pan-tropical biomass map using multiple reference datasets. Glob. Chang. Biol. 22(4):1406-20.

Bagchi R, Swamy V, Latorre Farfán JP, Terborgh J, Vela C, Pitman N, Galiano W. 2018. Defaunation increases the spatial clustering of lowland Western Amazonian tree communities. Journal of Ecology 106:1470-82.

Baker T, Del Castillo D, Honorio E, Lawson I, Martín M, Montoya M y Roucoux K. 2020. The challenges for achieving conservation and sustainable development within the wetlands of the Pastaza-Marañon basin, Peru. En Chirif
Tirado A, ed., Peru: Deforestation in Times of Climate Change. Lima: IWGIA. 155-74.

Benavides J y Vitt D. 2014. Response curves and the environmental limits for peat-forming species in the northern Andes. Plant Ecology 215:937-52.

Bhomia R, Van Lent J, Grandez J, Hergoualc'h K, Honorio E, Murdiyarso D. 2019. Impacts of Mauritia flexuosa degradation on the carbon stocks of freshwater peatlands in the PastazaMarañón river basin of the Peruvian Amazon. Mitig Adapt Strateg Glob Change 24:645-68.

Bodmer R, Puertas P, García J, Días D y Reyes C. 1999. Game animals, palms, and people of the flooded forests: Management considerations for the Pacaya-Samiria National Reserve, Peru. Advances in Economic Botany 13 (Várzea: Diversity, development, and conservation of Amazonia's whitewater floodplains):217-31.

Chimner R. 2019. Reservas de carbono y ciclos de carbono de las turberas andinas. Presentación. Simposio Nacional sobre la Gestión Sostenible de las Turberas Peruanas. Lima, Perú. https://www.slideshare.net/CIFOR/ reservas-de-carbono-y-ciclo-de-carbono-de-lasturberas-andinas

Chimner R, Bourgeau-Chávez L, Grelik S, Hribljan J, Planas A, Polk M, Lilleskov E, Fuentealba B. 2019. Mapping mountain peatlands and wet meadows using multi-date, multi-sensor remote sensing in the Cordillera Blanca, Peru. Wetlands 39:1057-67. https://doi.org/10.1007/s13157019-01134-1

Cooper DJ, Wolf EC, Colson C, Vering W, Granda A y Meyer M. 2010. Alpine peatlands of the Andes, Cajamarca, Peru. Arctic Antarctic and Alpine Research 42:19-33.

Draper F, Coronado E, Roucoux K, Lawson I, Pitman N, Fine P, Phillips O, Montenegro L, Sandoval E, Mesones I, García-Villacorta R, Arévalo F y Baker T. 2017. Peatland forests are the least diverse tree communities documented in Amazonia, but contribute to high regional beta-diversity. Ecography 41:1256-69. 
Draper F, Roucoux K, Lawson I, Mitchard E, Honorio E, Lähteenoja O, Torres L, Valderrama E, Zaráte R y Baker T. 2014. The distribution and amount of carbon in the largest peatland complex in Amazonia. Environmental Research Letters 9:124017-29.

Drösler M, Verchot L, Freibauer A, Pan G, Evans C, Bourbonniere R, Alm J, Page S, Agus F, Hergoualc'h K, Couwenberg J, et al. 2014. Drained inland organic soils (chaper 2). En Hiraishi T, Krug T, Tanabe K, Srivastava N, Jamsranjav B, Fukuda M y Troxler T, eds., 2013 Supplement to the 2006 IPCC Guidelines for National Greenhouse Gas Inventories: Wetlands. Suiza: IPCC.

Falen L y Honorio E. 2018. Evaluación de las técnicas de aprovechamiento de frutos de aguaje (Mauritia flexuosa L.f.) en el distrito de Jenaro Herrera, Loreto, Perú. Folia Amazónica 27:131-50.

Freitas L, Zárate R, Bardales R y Del Castillo D. 2019. Efecto de la densidad de siembra en el desarrollo vegetativo del aguaje (Mauritia flexuosa L.f.) en plantaciones forestales. Revista Peruana de Biología 26(2):227-34.

García E y Otto M. 2015. Caracterización ecohidrológica de humedales altoandinos usando imágenes de satélite multitemporales en la cabecera de cuenca del río Santa, Áncash, Perú. Ecología Aplicada 14(2):115-125.

Gaveau D, Salim M, Hergoaualc'h K, Locatelli B, Sloan S, Wooster M, Marlier M, Molidena E, Yaen H, DeFries R, Verchot L, Murdiyarso D, Nasi R, Holmgren P y Sheil D. 2014. Major atmospheric emissions from peat fires in Southeast Asia during non-drought years: Evidence from the 2013 Sumatran fires. Sci Rep 4, 6112.

Gilmore MP, Endress BA y Horn CM. 2013. The socio-cultural importance of Mauritia flexuosa palm swamps (aguajales) and implications for multi-use management in two Maijuna communities of the Peruvian Amazon. $J$ Ethnobiol Ethnomed 9(29). https://doi. org/10.1186/1746-4269-9-29

Gloor M, Brienen R, Galbraith D, Feldpausch T, Schongart J, Guyot J, Espinoza J, Lloyd J, Phillips O. 2013. Intensification of the Amazon hydrological cycle over the last two decades. Geophysical Research Letters 40:1729-33.

Griffis T, Roman T, Wood J, Deventer J, Fachin L, Rengifo J, Del Castillo D, Lilleskov E, Kolka R, Chimner R, Del Águila J, et al. 2020. Hydrometeorological sensitivities of net ecosystem carbon dioxide and methane exchange of an Amazonian palm swamp peatland. Agricultural and Forest Meteorology 295.

Griscom B, Adams J, Ellis P, Houghton R, Lomax G, Miteva D, Schlesinger WH, Shoch D, Siikamäki JV, Smith P, et al. 2017. Natural climate solutions. Proceedings of the National Academy of Sciences of the United States of America 114:11645-50.

[GTM-NDC] Grupo de Trabajo Multisectorial para la Implementación de las NDC. 2018. Informe final. http://www.minam.gob.pe/ cambioclimatico/gtm/

Gumbricht T, Román-Cuesta R, Verchot L, Herold M, Wittmann F, Householder E, Herold N y Murdiyarso D. 2017. An expert system model for mapping tropical wetlands and peatlands reveals South America as the largest contributor. Global Change Biology 23:3581-99.

Harmon D. 1996. Losing species, losing languages: Connections between biological and linguistic diversity. Southwest Journal of Linguistics (15):89-108.

Hergoualc'h K, Carmenta R, Atmadja S, Martius C, Murdiyarso D y Purnomo H. 2018. Managing peatlands in Indonesia: Challenges and opportunities for local and global communities. CIFOR Infobrief 205.

Hergoualc'h K, Dezzeo N, Verchot C, Van Lent J, Del Águila J, López M. 2020. Spatial and temporal variability of soil $\mathrm{N}_{2} \mathrm{O}$ and $\mathrm{CH}_{4}$ fluxes along a degradation gradient in a palm swamp peat forest in the Peruvian Amazon. Global Change Biology. doi:10.1111/gcb.15354

Hergoualc'h K, Gutiérrez-Vélez V, Menton M y Verchot L. 2017. Characterizing degradation of palm swamp peatlands from space and on the ground: An exploratory study in the Peruvian Amazon. Forest Ecology and Management 393:63-73. Traducción al español disponible en: http://www.cifor.org/knowledge/ publication/6652/

Hiraishi T, Krug T, Tanabe K, Srivastava N, Fukuda M, Troxler T y Jamsranjav B. 2013. Supplement to the 2006 IPCC Guidelines for National Greenhouse Gas Inventories: Wetlands. Suiza: IPCC. Fecha de acceso: 13 de febrero de 2018. http://www.ipcc-nggip.iges.or.jp/public/ wetlands/pdf/Wetlands_Supplement_Entire_ Report.pdf

Horn C, Gilmore M y Endress B. 2012. Ecological and socio-economic factors influencing aguaje (Mauritia flexuosa) resource management in 
two indigenous communities in the Peruvian Amazon forest. Ecol. Manag. 267:93-103.

Horn C, Paredes V, Gilmore M, Endress B. 2018. Spatio-temporal patterns of Mauritia flexuosa fruit extraction in the Peruvian Amazon: Implications for conservation and sustainability. Applied Geography 97:98-108.

Hribljan J, Suárez E, Bourgeau-Chávez L, Endres S, Lilleskov E, Chimbolema S, Wayson C, Serocki E y Chimner R. 2017. Multidate, multisensor remote sensing reveals high density of carbon rich mountain peatlands in the páramo of Ecuador. Global Change Biology 23(12):5412-25. https://doi.org/10.1111/ gcb. 13807

Huamán Y, Moreira-Turq P, Willems B, Espinoza R, Apaéstegui J y Llanos R. 2017. Cambios en la acumulación de carbono en bofedales andinos durantes los últimos 3000 años AP. En Libro de resúmenes. I Congreso Peruano de Humedales.

[INAIGEM] Instituto Nacional de Investigación en Glaciares y Ecosistemas de Montaña. 2017. Informe de la situación de los glaciares y ecosistemas de montaña.

[IPCC] Intergovernmental Panel on Climate Change. 2014: Climate Change 2014: Synthesis Report. Contribution of Working Groups I, II and III to the Fifth Assessment Report of the Intergovernmental Panel on Climate Change [Core Writing Team, Pachauri RK y Meyer LA, eds.]. Ginebra: IPCC. https://ar5-syr.ipcc. ch/topic_summary.php

Janovec J, Householder E, Tobler M, Valega R, Von May R, Araujo J, Zelski S, Shearer C, Jiménez M, Wells J, Chambi B, Herrera F y Pérez M. 2013. Humedales de Madre de Dios, Perú: impactos y amenazas en aguajales y cochas. Lima, Perú: WWF.

Kahn F, Mejía K, Moussa F y Gómez D. 1993. Mauritia flexuosa, la más acuática de las palmeras amazónicas. En Kahn F, León B y Young K. Las plantas vasculares en las aguas continentales del Perú. Tomo 75, capítulo VIII. 287-308.

Lähteenoja O y Page S. 2011. High diversity of tropical peatland ecosystem types in the PastazaMaranon basin, Peruvian Amazonia. Journal of Geophysical Research-Biogeosciences 116.

Lähteenoja O, Reátegui Y, Rasanen M, Del Castillo D, Oinonen M y Page S. 2012. The large Amazonian peatland carbon sink in the subsiding Pastaza-Marañon foreland basin, Peru. Global Change Biology 18:164-78.
Lähteenoja O, Ruokolainen K, Schulman L y Oinonen M. 2009. Amazonian peatlands: An ignored C sink and potential source. Global Change Biology 15:2311-20.

Lilleskov E, McCullough K, Hergoualc'h K, Del Castillo D, Chimner R, Murdiyarso D, Kolka R, Bourgeau R, Hribljan J, Del Águila J y Wayson C. 2017. Is Indonesian peatland loss a cautionary tale for Peru? A two-country comparison of the magnitude and causes of tropical peatland degradation. Mitigation and Adaptation Strategies for Global Change 24:591-623. Infobrief en español disponible en: https://www.cifor.org/knowledge/ publication/7491

Loh J y Harmon D. 2014. Biocultural Diversity: Threatened Species, Endangered Languages. Países Bajos: WWF-Netherlands.

Machuca D. 2018. Efectos de la extracción de turba en un sistema socioecológico andino: bofedales de Carampoma - Lima [tesis para optar el título de licenciado en Geografía y Medio Ambiente]. Lima: Pontificia Universidad Católica del Perú.

Maldonado M. 2010. Comportamiento de la vegetación de bofedales influenciados por actividades antrópicas [tesis de maestría]. Lima: Pontificia Universidad Católica del Perú.

Maldonado Fonkén M. 2014. An introduction to the bofedales of the Peruvian high Andes. Mires Peat 15:1-13.

Martín M, Núñez C, Fabiano E, Del Águila M, Shulz C, Laurie N, Sanjurjo J, Davies A, Roucoux K, Lawson I y Andueza L. 2019. Urarina: identidad y memoria en la cuenca del rio Chambira. Iquitos: IIAP/MINAM; PUCP; University of Saint Andrews.

Medrano R, Chupan L y Vila M. 2012. Almacenamiento de carbono en especies predominantes de flora en el lago Chinchaycocha. Apuntes de Ciencia y Sociedad 2(2):110-117.

Melack JM, Hess LL, Gastil M, Forsberg BR, Hamilton SK, Lima IB y Novo EM. 2004. Regionalization of methane emissions in the Amazon Basin with microwave remote sensing. Global Change Biology 10:530-44.

Miettinen J, Hooijer A, Shi C, Tollenaar D, Vernimmen R, Liew S, Malins C y Page S. 2012. Extent of industrial plantations on Southeast Asian peatlands in 2010 with analysis of historical expansion and future projections. GCB Bioenergy 4(6):908-18. https://doi.org/10.1111/j.17571707.2012.01172.x 
[MINAM] Ministerio del Ambiente del Perú. 2019. Inventario Nacional de Gases de Efecto Invernadero (INGEI) 2014.

[MINAM] Ministerio del Ambiente del Perú. 2018a. Decreto Supremo que aprueba la categorización de la Zona Reservada Yaguas como Parque Nacional Yaguas. Decreto Supremo No. 001-2018-MINAM. Lima: MINAM.

[MINAM] Ministerio del Ambiente del Perú. 2018b. Mapa de ecosistemas del Perú. Memoria descriptiva. https://sinia.minam.gob.pe/mapas/ mapa-nacional-ecosistemas-peru

[MINAM] Ministerio del Ambiente del Perú. 2016. Estrategia Nacional sobre Bosques y Cambio Climático. Decreto Supremo N. ${ }^{\circ}$ 007-2016-MINAM. http:// www.bosques.gob.pe/archivo/ff3f54_ ESTRATEGIACAMBIOCLIMATICO 2016_ok.pdf

[MINAM] Ministerio del Ambiente del Perú. 2015. Estrategia Nacional de Humedales. Decreto Supremo N.o 004-2015-MINAM.

Noriega C. 2012. Nueva Cajamarca: una ciudad andina en la Amazonía. En Lo urbano en el Perú. Serie Perú Hoy n.o 22. 269-290.

Oktarita S, Hergoualc'h K, Anwar S y Verchot $\mathrm{LV}, 2017$. Substantial $\mathrm{N}_{2} \mathrm{O}$ emissions from peat decomposition and $\mathrm{N}$ fertilization in an oil palm plantation exacerbated by hotspots. Environ. Res. Lett. 12(10).

Padoch C. 1988. Aguaje (Mauritia flexuosa L.f.) in the economy of Iquitos, Peru. En Balick M, ed., The Palm-Tree of Life: Biology, Utilization and Conservation. Advances in Economic Botany V6. Nueva York: Botanical Garden.

Peres C, Thaise E, Schietti J y Levi T. 2016. Dispersal limitation induces long-term biomass collapse in overhunted Amazonian forests. Proceedings of the National Academy of Sciences 113(4):892-7.

Planas A, Chimner R, Hribljan J, Lilleskov E y Fuentealba B. 2020. The effect of water table levels and short-term ditch restoration on mountain peatland carbon cycling in the Cordillera Blanca, Peru. Wetlands Ecol. Manage 28:51-69.

Pröpper M y Haupts F, 2014. The culturality of ecosystem services. Emphasizing process and transformation. Ecological Economics 108:28-35.

Ramsar. 2018. Lineamientos para inventarios de turberas tropicales a fin de facilitar su designación como sitios Ramsar. Nota Informativa de Ramsar 9. https:// www.ramsar.org/sites/default/files/documents/ library/bn9_peatland_inventory_s.pdf
Ramsar. 2002. Resolución VIII.17. Lineamientos para la acción mundial sobre las turberas. https:/www.ramsar.org/sites/default/files/ documents/pdf/res/key_res_viii_17_s.pdf

República del Perú. 2017. Ley no. 30670. Ley que declara de necesidad pública e interés nacional la construcción de la carretera IquitosSaramiriza para su interconexión con la costa norte. Lima: RoP. https://busquedas. elperuano.pe/normaslegales/ley-que-declarade-necesidad-publica-e-interes-nacional-la-cley-n-30670-1572534-2/

República del Perú. 2016. Ley no. 30215. Ley de Mecanismos de Retribución por Servicios Ecosistémicos. Lima: RoP. https://busquedas. elperuano.pe/normaslegales/apruebanreglamento-de-la-ley-n-30215-ley-demecanismos-de-decreto-supremo-n-009-2016minam-1407244-4/

República del Perú. 2015. Contribución prevista $y$ determinada a nivel nacional (INDC) de la República del Perú. https://www4.unfccc. int/sites/ndcstaging/PublishedDocuments/ Peru\%20First/iNDC\%20Per\%C3\%BA\%20 castellano.pdf

República del Perú. 1997. Ley no. 26834. Ley de Areas Naturales Protegidas. Lima: RoP. http:// www.leyes.congreso.gob.pe/Documentos/ Leyes/26834.pdf

Román-Cuesta R, Salinas N, Asbjornsen H, Oliveras I, Huamán V, Gutiérrez Y, Puelles L, Kala J, Yabar D, Rojas M, Astete R, et al. 2011. Implications of fires on carbon budgets in Andean cloud montane forest: The importance of peat soils and tree resprouting. Forest Ecology and Management 261(11):1987-97.

Ross N. 2002. Lacandon Maya intergenerational change and the erosion of folk biological knowledge. En Stepp JR, Wyndham FS y Zarger R., eds., Ethnobiology and Biocultural Diversity. Atenas, Grecia: International Society of Ethnobiology.

Roucoux K, Lawson I, Baker T, Honorio E, Gosling W y Lahteenoja O. 2013. Vegetation development in an Amazonian peatland. Palaeogeography, Palaeoclimatology, Palaeoecology 374:242-55.

Roucoux K, Lawson I, Baker T, Torres D, Draper FC, Lahteenoja O, Gilmore MP, Honorio E, Kelly TJ, Mitchard ET y Vriesendorp CF. 2017. Threats to intact tropical peatlands and opportunities for their conservation. Conservation Biology 31:1283-92. 
Salvador F y Cano A. 2002. Lagunas y oconales: los humedales del trópico andino. Cuadernos de Biodiversidad 11:4-9.

Salvador F, Monerris J y Rochefort L. 2014. Peatlands of the Peruvian Puna ecoregion: Types, characteristics and disturbance. Mires and Peat 15.

Sánchez M, Chimner R, Hribljan J, Lilleskov E y Suárez E. 2017. Carbon dioxide and methane fluxes in grazed and undisturbed mountain peatlands in the Ecuadorian Andes. Mires and Peat 19:1-18. doi:10.19189/MaP.2017. OMB.277

Saatchi S, Houghton RA, Dos Santos Alvalá R, Soares J y Yu Y. 2007. Distribution of aboveground live biomass in the Amazon basin. Global Change Biology 13:816-37. doi:10.1111/j.1365-2486.2007.01323.x

Schulz C, Martín M, Pérez C, Villacorta M, Laurie N, Lawson I y Roucoux K. 2019. Peatland and wetland ecosystems in Peruvian Amazonia: Indigenous classifications and perspectives. Ecology and Society 24.

[SERNANP] Servicio Nacional de Áreas Naturales Protegidas por el Estado. 2020. Reserva Nacional Pacaya Samiria. Fecha de acceso: 5 de julio de 2020. https://www. sernanp.gob.pe/aguaje-pacaya

Swindles G, Morris P, Whitney B, et al. 2017. Ecosystem state shifts during long-term development of an Amazonian peatland. Glob. Change Biol. 24:738-57.

Tropical Forest Alliance. 2020. International promotion of Peru's "tree of life" fruit to generate income for indigenous communities and protect Peru's valuable Amazonian forest [nota de prensa]. Fecha de acceso: 10 de septiembre de 2020. https://www.tropicalforestalliance.org/

Van Lent J, Hergoualc'h K, Verchot L, Oenema O y Van Groenigen J. 2019. Greenhouse gas emissions along a peat swamp forest degradation gradient in the Peruvian Amazon: Soil moisture and palm roots effects. Mitig Adapt Strateg Glob Change 24:625-43.

Verzijl A y Guerrero S. 2013. The system nobody sees: Irrigated wetland management and alpaca herding in the Peruvian Andes. Mountain Research and Development 33(3):280-93.

Wang S, Zhuang Q, Lähteenoja O, Draper F y Cadillo-Quiroz H. 2018. Potential shift from a carbon sink to a source in Amazonian peatlands under a changing climate. Proc. Natl. Acad. Sci. U.S.A. 115:12407-12.

Xu J, Morris P, Liu, J y Holden J. 2018. Peatmap: Refining estimates of global peatland distribution based on a meta-analysis. Catena 160:134-140.

Yu Z, Beilman D, Frolking S, MacDonald G, Roulet N, Camill P y Charman D. 2012. Peatlands and their role in the global carbon cycle. Eos 92(12).

Ziegler G. 2020. Arrasar la tierra: una comunidad resiste el tráfico de humedales. Ojo Público. Fecha de acceso: 15 de julio de 2020. https:// ojo-publico.com/1946/arrasar-la-tierra-eltrafico-de-humedales-en-sierra-de-lima 

Los Documentos Ocasionales de CIFOR contienen resultados de investigación relevantes para el manejo forestal tropical. Su contenido es revisado por pares interna y externamente.

- El Perú es uno de los países del trópico más ricos en turberas. Cuenta con ellas en sus tres regiones, con una preponderancia marcada en la Amazonía. Sus turberas proveen importantes servicios ecosistémicos, como el almacenamiento de inmensas cantidades de carbono, la fijación de dióxido de carbono, una biodiversidad única, la regulación hídrica a nivel local y regional, y el suministro de medios de subsistencia y valores culturales para las poblaciones locales.

- Las turberas del país están siendo deterioradas por actividades antropogénicas que incluyen el desarrollo de infraestructura y la extracción de recursos (p. ej., petróleo, minerales), y usos o prácticas no sostenibles de intensidad variable (p. ej., sobrepastoreo, extracción de turba, tala de palmeras, sobrecaza) que las amenazan e incrementan su vulnerabilidad. De igual manera, los cambios climáticos comprometen su estabilidad.

- El marco normativo peruano incluye normas e instrumentos para una gestión sostenible de los humedales, pero falta desarrollar regulaciones específicas para las turberas. Entre los avances recientes está la elaboración de una definición normativa nacional del término "turbera"; sin embargo, aún se requiere su inclusión explícita en políticas relativas al cambio climático, como REDD+ y las Contribuciones Nacionalmente Determinadas (NDC, por sus siglas en inglés).

- Existe una falta fundamental de investigación científica sobre las turberas peruanas. En particular, se requiere cartografiarlas, inventariarlas y caracterizar sus propiedades ecológicas y sus valores económicos y sociales. También es esencial identificar y revalorar los conocimientos que las comunidades indígenas ponen en práctica para gestionarlas de manera sostenible.

- Las oportunidades para la conservación y buena gestión de estos ecosistemas claves son diversas e incluyen, por ejemplo, la consolidación de los mecanismos de pago por servicios ecosistémicos, la implementación de planes de manejo sostenible de recursos por las poblaciones locales, la extensión de las áreas naturales protegidas (ANP) y el reconocimiento de los derechos de tenencia de las comunidades.

\begin{tabular}{|l|ll}
\hline & $\begin{array}{l}\text { Esta investigación fue realizada por CIFOR como parte del Programa de Investigación de CGIAR sobre } \\
\text { Bosques, Árboles y Agroforestería (FTA). El FTA es el programa de investigación para el desarrollo } \\
\text { más }\end{array}$ \\
INVESTIGACIÓN SOBRE & $\begin{array}{l}\text { más grande del mundo, dedicado a mejorar el papel de bosques, árboles y la agroforestería para el } \\
\text { BOSques, Árboles y } \\
\text { desarrollo sostenible, la seguridad alimentaria, y frente al cambio climático. CIFOR dirige el programa }\end{array}$ \\
Agroforestería & FTA en asociación con Bioversity International, CATIE, CIRAD, ICRAF, INBAR y TBI. \\
La investigación del Programa FTA cuenta con el apoyo del Fondo Fiduciario del CGIAR: cgiar.org/funders/
\end{tabular}

cifor.org

forestsnews.cifor.org desarrollando las capacidades de sus socios y dialogando activamente con todos los actores involucrados, para informar sobre las políticas y las prácticas que afectan a los bosques y a las personas. CIFOR es un centro de investigación CGIAR y lidera su Programa de Investigación sobre Bosques, Árboles y Agroforestería (FTA por sus siglas en inglés). Nuestra sede central se encuentra en Bogor, Indonesia, y contamos con oficinas en Nairobi, Kenia; Yaundé, Camerún; Lima, Perú, y Bonn, Alemania. 